\title{
REGULARITY AND ENTROPY SOLUTIONS OF SOME ELLIPTIC EQUATIONS
}

\section{ALIZADEH AND M. ALIMOHAMMADY}

Received 03 March, 2018

\begin{abstract}
Here, we would study regularity of solutions and existence at least one entropy solution for $L^{1}$-data and duality solution. This result would improve some results of Laplacian differential equations.
\end{abstract}

2010 Mathematics Subject Classification: 35B65; 35J55

Keywords: Fourier transformation, regularity, duality solutions, entropy solutions

\section{INTRODUCTION}

First, we summarize some result notions of Schwratz space and tempered distributions. The schwartz space $\delta\left(\mathbb{R}^{n}\right)$ is a topological vector space of all $f: \mathbb{R}^{n} \rightarrow \mathbb{C}$ such that $f \in C^{\infty}\left(\mathbb{R}^{n}\right)$ and $x^{\alpha} \partial^{\beta} f(x)$ is bounded. For every pair of multi-induces $\alpha, \beta \in \mathbb{N}^{n}$, we set

$$
\|\left.|f|\right|_{\alpha, \beta}:=\sup _{x}\left|x^{\alpha} \partial^{\beta} f\right|
$$

which induces a family of semi-norms on $\delta\left(\mathbb{R}^{n}\right)$. A tempered distribution is a continuous linear functional $T: \delta\left(\mathbb{R}^{n}\right) \rightarrow \mathbb{C}$ and $\boldsymbol{\delta}^{\prime}\left(\mathbb{R}^{n}\right)$ is the space of all tempered distributions. $\mathscr{D}\left(\mathbb{R}^{n}\right)$ is the space $C_{c}^{\infty}\left(\mathbb{R}^{n}\right)$ endowed with the topology in which $f_{n} \rightarrow 0$ means that, there is a compact set $K$; such that $\operatorname{Supp}_{n} \subseteq K(n=1,2, \ldots)$ and for each $\alpha \in \mathbb{N}^{n}, D^{\alpha} f_{n} \rightarrow 0$ uniformly.

The fourier transform of a function $f \in \mathcal{S}\left(\mathbb{R}^{n}\right)$ is the function $\widehat{f}: \mathbb{R}^{n} \rightarrow \mathbb{C}$ defined by

$$
\widehat{f}(k)=\frac{1}{(2 \pi)^{\frac{n}{2}}} \int_{\mathbb{R}^{n}} f(x) e^{-i k x} d x .
$$

It is well known that

$1)^{\Upsilon}: \delta\left(\mathbb{R}^{n}\right) \rightarrow \delta\left(\mathbb{R}^{n}\right)$ is continuous one to one.

2) $\widehat{\partial^{\alpha} f}(k)=(i k)^{\alpha} \widehat{f}(k)$.

3) $\widehat{(-i x)^{\beta} f}(k)=\partial^{\beta} \widehat{f}(k)$.

For regularity of Laplacian, Ma and Thompson [8], Ma [7] proved regularity, where 
$f \in C[0,1]$. Moreover, Lee and Sim [6] proved it but for $f \in L^{1}(0,1)$. Recently, interior regularity have studied by many mathematician:

M. Cozzi [4] studied regularity theory of weak solutions for the second order linear elliptic differential equations $-\operatorname{div}(A(.) \nabla u)=f$ in $\Omega$, where $\Omega$ is an open bounded subset of $\mathbb{R}^{n}$ and $A=\left[a_{i j}\right]$ is $n \times n$ matrix uniformly elliptic, $a_{i j} \in C_{\text {loc }}^{0,1}(\Omega)$ and $f \in L^{2}(\Omega)$. In fact, it is proved that for any $\Omega^{\prime} \subset \subset \Omega,\|u\|_{H^{2}(\Omega)} \leq C\left(\|u\|_{L^{2}(\Omega)}+\right.$ $\left.\|f\|_{L^{2}(\Omega)}\right)$. Moreover, it was shown the interior $H^{2 s-\epsilon}$ regularity for weak solutions of some linear elliptic differential equations.

J. Siljander, J. M. Urbano [11] studied the Serrin-type interior regulatity result.

$$
u \in L_{l o c}^{2+\epsilon}\left(\Omega_{T}\right) \Rightarrow \text { regularity }
$$

for a weak solution in the energy space $L_{t}^{\infty} L_{x}^{2}$ satisfying in appropriate vorticity estimates for

$$
\partial_{t} u+(u . \nabla) u+\nabla p=0 \text { and } \operatorname{div} u=0 .
$$

S. Gustafson and co authors [5] gave an interior regularity criteria for suitable weak solutions of the 3D Navier-Stokes equations. In fact they considered the regularity problem for a suitable weak solution $(u, p): \Omega \times I \rightarrow \mathbb{R}^{3} \times \mathbb{R}$ of three-dimensional incompressible Navier-Stokes equations (NS)

$$
\begin{cases}u_{t}-\Delta u+(u . \nabla) u+\nabla p=f & \text { in } \Omega \\ \operatorname{div} u=0 & \text { in } \Omega \times I\end{cases}
$$

and proved $u \in L^{\infty}\left(Q_{z, r}\right)$ for some $B_{x, r} \times\left(t-r^{2}, t\right)=Q_{z, r} \subseteq \Omega \times I, r>0$.

These brand of problems have potential applications to the modeling of combustion, thermal explosions, nonlinear heat generation, gravitational equilibrium of polytropic stars, glaciology, non-Newtonian fluids, and the flow through porous media.

In this paper, we would study the regular property of

$$
-\Delta_{\alpha} u+\lambda u=f
$$

in $\mathscr{D}^{\prime}(\Omega)$, where we define $\Delta_{\alpha}:=\alpha_{1} \frac{\partial^{2}}{\partial x_{1}^{2}}+\ldots+\alpha_{n} \frac{\partial^{2}}{\partial x_{n}^{2}}$ and $\alpha=\left(\alpha_{1}, \ldots, \alpha_{n}\right),\left(\alpha_{i}>\right.$ $0, \forall i)$, our aim is to show $u \in C^{\infty}\left(\mathbb{R}^{n}\right)$.

\section{Results}

Here, we use of $\approx$ for equivalent norms.

Lemma 1. Suppose that $m \in \mathbb{Z}, \lambda>0$ and let $u, f \in \mathcal{S}^{\prime}\left(\mathbb{R}^{n}\right)$ satisfy (1.1) as distributions. If $f \in W^{m, 2}\left(\mathbb{R}^{n}\right)$, then $u \in W^{m+2,2}\left(\mathbb{R}^{n}\right)$ and there exists a constant $C$ such that $\|u\|_{W^{m+2,2}} \leq C\|f\|_{W^{m, 2}}$.

Proof. Taking the Fourier transform of (1.1), we have

$$
\mathscr{F}\left(-\alpha_{1} u_{x_{1} x_{1}}-\ldots-\alpha_{n} u_{x_{n} x_{n}}+\lambda u\right)=\mathscr{F}(f),
$$




$$
\left(\alpha_{1} \xi_{1}^{2}+\ldots+\alpha_{n} \xi_{n}^{2}+\lambda\right) \mathcal{F}(u)=\mathscr{F}(f) .
$$

From theorem 5.2.3 of [3] for any $m \in \mathbb{Z}$ and $a, b>0$ we note to the following equivalent norms:

$$
\begin{gathered}
W^{m, 2}\left(\mathbb{R}^{n}\right):=\left\{u \in \mathcal{S}^{\prime}\left(\mathbb{R}^{n}\right) ; \mathcal{F}^{-1}\left[\left(a+b|\xi|^{2}\right)^{\frac{m}{2}} \mathcal{F}(u)\right] \in L^{2}\left(\mathbb{R}^{n}\right)\right\} \\
\|u\|_{W^{m, 2}} \approx\left\|\mathcal{F}^{-1}\left[\left(a+b|\xi|^{2}\right)^{\frac{m}{2}} \mathcal{F}(u)\right]\right\|_{L^{2}}, \quad u \in W^{m, 2}\left(\mathbb{R}^{n}\right) .
\end{gathered}
$$

By using Parseval theorem

$$
\left\|\mathcal{F}^{-1}\left[\left(a+b|\xi|^{2}\right)^{\frac{m}{2}} \mathcal{F}(u)\right]\right\|_{L^{2}}=\left\|\left(a+b|\xi|^{2}\right)^{\frac{m}{2}} \mathcal{F}(u)\right\|_{L^{2}} .
$$

Thus,

$$
\left\|\left(a+b|\xi|^{2}\right)^{\frac{m}{2}} \mathcal{F}(u)\right\|_{L^{2}} \approx\left\|\left(\alpha_{1} \xi_{1}^{2}+\ldots+\alpha_{n} \xi_{n}^{2}+\lambda\right)^{\frac{m}{2}} \mathcal{F}(u)\right\|_{L^{2}}
$$

and

$$
\left(\alpha_{1} \xi_{1}^{2}+\ldots+\alpha_{n} \xi_{n}^{2}+\lambda\right)^{\frac{m+2}{2}} \mathcal{F}(u)=\left(\alpha_{1} \xi_{1}^{2}+\ldots+\alpha_{n} \xi_{n}^{2}+\lambda\right)^{\frac{m}{2}} \mathcal{F}(f)
$$

so the result follows from (2.1).

We now consider the case of a general domain $\Omega$

Theorem 1. Suppose that $\lambda \in \mathbb{R}$ and $u, f \in \mathscr{D}^{\prime}(\Omega)$ satisfy the equation (1.1) in $\mathscr{D}^{\prime}(\Omega)$.

(i) If $f \in W_{\text {loc }}^{m, 2}(\Omega)$ and $u \in W_{\text {loc }}^{n, 2}(\Omega)$ for some $m \geq 0$ and $n \in \mathbb{Z}$, then $u \in W_{\text {loc }}^{m+2,2}(\Omega)$ and for every $\Omega_{2} \subset \subset \Omega_{1} \subset \subset \Omega$, there exists a constant $C$ (depending only on $m, \Omega_{2}$ and $\left.\Omega_{1}\right)$ such that $\|u\|_{W^{m+2,2}\left(\Omega_{2}\right)} \leq C\left(\|f\|_{W^{m, 2}\left(\Omega_{1}\right)}+\right.$ $\left.\|u\|_{W^{n, 2}\left(\Omega_{1}\right)}\right)$.

(ii) If $f \in C^{\infty}(\Omega)$ and $u \in W_{\text {loc }}^{n, 2}(\Omega)$ for some $n \in \mathbb{Z}$ then $u \in C^{\infty}(\Omega)$.

Proof. We proceed in two steps.

Step 1: Consider $M^{\prime \prime} \subset \subset M^{\prime} \subset \subset \Omega$ and $k \in \mathbb{Z}$. If $u \in W^{k, 2}\left(M^{\prime}\right)$ and $f \in W^{k-1,2}\left(M^{\prime}\right)$ solve the equation (1.1) in $\mathscr{D}^{\prime}(\Omega)$, thus, $u \in W^{k+1,2}\left(M^{\prime \prime}\right)$ and there exists $C$ such that $\|u\|_{W^{k+1,2}\left(M^{\prime \prime}\right)} \leq C\left(\|f\|_{W^{k-1,2}\left(M^{\prime}\right)}+\|u\|_{W^{k, 2}\left(M^{\prime}\right)}\right)$. To show this, consider $\rho \in C_{c}^{\infty}\left(\mathbb{R}^{\mathrm{m}}\right)$ such that $\rho \equiv 1$ on $M^{\prime \prime}$ and supp $\rho \subset M^{\prime}$ and define $v \in \mathscr{D}^{\prime}\left(\mathbb{R}^{n}\right)$ by $v=\rho u$, i.e.

$$
(v, \varphi)_{D^{\prime}\left(\mathbb{R}^{n}\right), D\left(\mathbb{R}^{n}\right)}=(u, \rho \varphi)_{D^{\prime}\left(M^{\prime}\right), D\left(M^{\prime}\right)} .
$$

Clearly $v \in W^{k, 2}\left(\mathbb{R}^{n}\right)$ and $\|v\|_{W^{k, 2}\left(\mathbb{R}^{n}\right)} \leq C\|u\|_{W^{k, 2}\left(M^{\prime}\right)}$.

$v$ solves the equation

$$
-\Delta_{\alpha} v+v=T_{1}+T_{2}+T_{3}
$$

in $\mathscr{D}^{\prime}\left(\mathbb{R}^{n}\right)$, where the distributions $T_{1}, T_{2}$, and $T_{3}$ are defined by

$$
\begin{gathered}
\left(T_{1}, \varphi\right)_{D^{\prime}\left(\mathbb{R}^{n}\right), \mathscr{D}\left(\mathbb{R}^{n}\right)}=(f+(1-\lambda) u, \rho \varphi)_{\mathscr{D}^{\prime}\left(M^{\prime}\right), \mathscr{D}\left(M^{\prime}\right),}, \\
\left(T_{2}, \varphi\right)_{D^{\prime}}\left(\mathbb{R}^{n}\right), \mathscr{D}\left(\mathbb{R}^{n}\right)=-\left(u, \Delta_{\alpha} \rho . \varphi\right)_{D^{\prime}\left(M^{\prime}\right), \mathscr{D}\left(M^{\prime}\right),}
\end{gathered}
$$




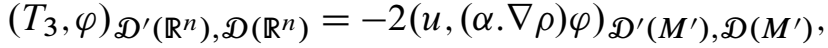

$$
\begin{aligned}
& \text { for every } \varphi \in C_{c}^{\infty}\left(\mathbb{R}^{n}\right) \text {, since }
\end{aligned}
$$

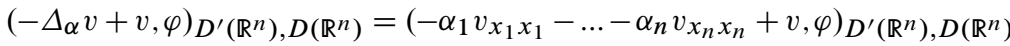

$$
\begin{aligned}
& =-\left(\alpha_{1}\left[\left(u, \rho_{x_{1} x_{1}} \varphi\right)_{D^{\prime}\left(M^{\prime}\right), D\left(M^{\prime}\right)}+2\left(u, \rho_{x_{1}} \varphi\right)_{D^{\prime}\left(M^{\prime}\right), D\left(M^{\prime}\right)}+\left(u_{x_{1} x_{1}}, \rho \varphi\right)_{D^{\prime}\left(M^{\prime}\right), D\left(M^{\prime}\right)}\right]\right. \\
& \left.+\ldots+\alpha_{n}\left[\left(u, \rho_{x_{n} x_{n}} \varphi\right)_{D^{\prime}\left(M^{\prime}\right), D\left(M^{\prime}\right)}+2\left(u, \rho_{x_{n}} \varphi\right)_{D^{\prime}\left(M^{\prime}\right), D\left(M^{\prime}\right)}+\left(u_{x_{n} x_{n}}, \rho \varphi\right)_{D^{\prime}\left(M^{\prime}\right), D\left(M^{\prime}\right)}\right]\right) \\
& +(u, \rho \varphi)_{D^{\prime}\left(M^{\prime}\right), D\left(M^{\prime}\right)}=\left(-\alpha_{1} u_{x_{1} x_{1}}-\ldots-\alpha_{n} u_{x_{n} x_{n}}+\lambda u, \rho \varphi\right)_{D^{\prime}\left(M^{\prime}\right), D\left(M^{\prime}\right)} \\
& -\left(u,\left(\alpha_{1} \rho_{x_{1} x_{1}}+\ldots+\alpha_{n} \rho_{x_{n} x_{n}}\right) \varphi\right)_{D^{\prime}\left(M^{\prime}\right), D\left(M^{\prime}\right)}-2\left(u,\left(\alpha_{1} \rho_{x_{1}}+\ldots+\alpha_{n} \rho_{x_{n}}\right) \varphi\right)_{D^{\prime}\left(M^{\prime}\right), D\left(M^{\prime}\right)} .
\end{aligned}
$$

Thus $T_{j} \in W^{k-1,2}\left(\mathbb{R}^{n}\right)$ and

$$
\left\|T_{j}\right\|_{W^{k-1,2}\left(\mathbb{R}^{n}\right)} \leq C\left(\|f\|_{W^{k-1,2}\left(M^{\prime}\right)}+\|u\|_{W^{k, 2}\left(M^{\prime}\right)}\right),
$$

for $j=1,2,3$. Applying (2.1) and lemma 1, we deduce $v \in W^{k+1,2}\left(\mathbb{R}^{n}\right)$ and $\|v\|_{W^{k+1,2}\left(\mathbb{R}^{n}\right)} \leq C\left(\|f\|_{W^{k-1,2}\left(M^{\prime}\right)}+\|u\|_{W^{k, 2}\left(M^{\prime}\right)}\right)$.

Step 2: (Conclusion) Without loss of generality, we may assume $n=-\ell \leq 0$. Let $\Omega_{2} \subset \subset \Omega_{1} \subset \subset \Omega$. Consider a family $\left(M_{j}\right)_{0 \leq j \leq m+\ell+1}$ of open subsets of $\Omega$, such that

$$
\Omega_{2}=M_{m+\ell+1} \subset \subset \ldots \subset \subset M_{0} \subset \subset \Omega_{1}
$$

(one constructs easily such a family). It follows from Step 1 that $u \in W^{-\ell+1,2}\left(M_{0}\right)$ and can

$$
\begin{gathered}
\|u\|_{W^{-\ell+1,2}\left(M_{0}\right)} \leq C\left(\|f\|_{W^{-\ell-1,2}\left(\Omega_{1}\right)}+\|u\|_{W^{-\ell, 2}\left(\Omega_{1}\right)}\right) \\
\leq C\left(\|f\|_{W^{m, 2}\left(\Omega_{1}\right)}+\|u\|_{W^{n, 2}\left(\Omega_{1}\right)}\right) .
\end{gathered}
$$

(2.3) and lemma 1 imply that $u \in W^{-\ell+2,2}\left(M_{1}\right)$ and

$$
\begin{gathered}
\|u\|_{W^{-\ell+2,2}\left(M_{1}\right)} \leq C\left(\|f\|_{W^{-\ell, 2}\left(M_{0}\right)}+\|u\|_{W^{-\ell+1,2}\left(M_{0}\right)}\right) \\
\leq C\left(\|f\|_{W^{m, 2}\left(\Omega_{1}\right)}+\|u\|_{W^{n, 2}\left(\Omega_{1}\right)}\right) .
\end{gathered}
$$

Iterating the above argument, $u \in W^{m+2,2}\left(M_{m+\ell+1}\right)=W^{m+2,2}\left(\Omega_{2}\right)$ and that there exists $C$ in which

$$
\|u\|_{W^{m+2,2}\left(\Omega_{2}\right)} \leq C\left(\|f\|_{W^{m, 2}\left(\Omega_{1}\right)}+\|u\|_{W^{n, 2}\left(\Omega_{1}\right)}\right) .
$$

Hence, property (i) satisfies since $\Omega_{1}$ and $\Omega_{2}$ are arbitrary. Property (ii) follows from Property (i) and $C^{\infty}(\Omega)=\bigcap_{m \geq 0} W_{l o c}^{m, 2}(\Omega)$.

Before paying to entropy solutions, we remember some notions:

Let $f, g$ and $q$ be functions in $L^{\infty}(\Omega), u$ and $v$ be the solutions of

$$
\begin{cases}-\operatorname{div}(A(x) \nabla u)+q(x) u=f & \text { in } \Omega \\ u=0 & \text { on } \partial \Omega\end{cases}
$$

and

$$
\begin{cases}-\operatorname{div}\left(A^{*}(x) \nabla v\right)+q(x) v=g & \text { in } \Omega \\ v=0 & \text { on } \partial \Omega\end{cases}
$$


respectively, where $A^{*}$ is the transposed matrix of $A$ and

$$
A(x) y . y \geq \alpha|y|^{2},|A(x)| \leq \beta
$$

for every $y \in \mathbb{R}^{\mathrm{m}}, 0<\alpha \leq \beta$. It is well known that (2.5) has a weak unique solution (Theorem 1.6.1 [1]). Since both $u$ and $v$ belong to $H_{0}^{1}(\Omega), u$ can be chosen as test function in the formulation of weak solution for $v$ and vice versa. One obtains

$$
\int f v=\int A(x) \nabla u \cdot \nabla v+\int q(x) u \cdot v=\int A^{*}(x) \nabla v \cdot \nabla u+\int q(x) v \cdot u=\int u g
$$

for every $f, g \in L^{\infty}(\Omega)$, where $u$ and $v$ solve the corresponding problems with data $f$ and $g$ respectively. $u, v \in L^{\infty}(\Omega)$ (Theorem 2.3 [9]), but we remark that the two integrals are well-defined also if $f \in L^{1}(\Omega)$ and $u \in L^{1}(\Omega)$ (always maintaining the assumption that $g$ and so $v$ is a bounded function). This fact inspired to Guido Stampacchia the following definition of solution for (2.5) if the datum is in $L^{1}(\Omega)$.

Definition 1. Suppose that $f \in L^{1}(\Omega)$. A function $u \in L^{1}(\Omega)$ is called a duality solution with datum $f$ if one has $\int u g=\int f v$, for every $g \in L^{\infty}(\Omega)$, where $v$ is the solution of

$$
\begin{cases}-\operatorname{div} v\left(A^{*}(x) \nabla v\right)+q(x) v=g & \text { in } \Omega \\ v=0 & \text { on } \partial \Omega\end{cases}
$$

Theorem 2 (Stampacchia, theorem 3.3 of [9]). For $f \in L^{1}(\Omega)$ there exists a unique duality solution with datum $f$. Furthermore, $u \in L^{q}(\Omega)$ for every $q<\frac{N}{N-2}$.

Remark 1. In special case if

$$
A=\left[\begin{array}{ccccccc}
\alpha_{1} & 0 & 0 & . & . & . & 0 \\
0 & \alpha_{2} & 0 & . & . & . & 0 \\
\cdot & & & & & & \cdot \\
. & & & & & & \cdot \\
. & & & & & & \cdot \\
0 & 0 & . & . & . & 0 & \alpha_{n}
\end{array}\right]
$$

and $q(x)=\lambda$, problems (2.5) and (2.6) change to

$$
\left\{\begin{array} { l l } 
{ - \Delta _ { \alpha } u + \lambda u = f } & { \text { in } \Omega } \\
{ u = 0 } & { \text { on } \partial \Omega }
\end{array} \quad \text { and } \quad \left\{\begin{array}{ll}
-\Delta_{\alpha} v+\lambda v=g & \text { in } \Omega \\
v=0 & \text { on } \partial \Omega
\end{array}\right.\right.
$$

Respectively.

Definition 2. For $k>0$, set

$$
T_{k}(s):=\max \{-k, \min \{s, k\}\}
$$

and

$$
\tau_{0}^{1,2}=\left\{u: \Omega \longrightarrow \mathbb{R} \text { measurable }: T_{k}(u) \in H_{0}^{1}(\Omega), \forall k>0\right\},
$$

It is well known that $\nabla T_{k}(u)=\nabla u \chi_{\{|u| \leq k\}}$. 
Lemma 2. Suppose that $u \in \tau_{0}^{1,2}(\Omega), q \in L^{\infty}(\Omega)$ and (2.7) valid. Then there is $\alpha>0$ in which

$$
\alpha \int_{\Omega}\left|\nabla T_{k}(u)\right|^{2} \leq \int_{\Omega} A(x) \nabla u . \nabla T_{k}(u)+\int_{\Omega} q(x) u T_{k}(u) .
$$

Proof. By (2.7)

$$
\begin{gathered}
\int_{\Omega} A(x) \nabla u \cdot \nabla T_{k}(u)+\int_{\Omega} q(x) u T_{k}(u) \geq \gamma \int_{\Omega}|\nabla u|^{2}+\int_{\Omega} q(x) u T_{k}(u) \\
\geq \gamma \int_{\Omega}|\nabla u|^{2}-\int_{\Omega}|q(x)||u|\left|T_{k}(u)\right|,
\end{gathered}
$$

since $\left|T_{k}(u)\right| \leq|u|,|q(x)| \leq b$ (almost every where) for a suitable $b>0$ and from Poincaré inequality

$$
\begin{gathered}
\gamma \int_{\Omega}|\nabla u|^{2}-\int_{\Omega}|q(x)||u|\left|T_{k}(u)\right| \geq \gamma \int_{\Omega}|\nabla u|^{2}-\int_{\Omega}|q(x)||u|^{2} \\
\geq \gamma \int_{\Omega}|\nabla u|^{2}-b C_{q} \int_{\Omega}|\nabla u|^{2}=\alpha \int_{\Omega}\left|\nabla T_{k}(u)\right|^{2}
\end{gathered}
$$

for $\alpha:=\gamma-b C_{q}$, where $C_{q}$ is multiplier in Poincaré inequality.

Definition 3. Suposse that $f \in L^{1}(\Omega)$. A function $u \in \tau_{0}^{1,2}(\Omega)$ is called an enteropy solution of (2.5) if

$$
\int_{\Omega} A(x) \nabla u \cdot \nabla T_{k}(u-\varphi)+\int_{\Omega} q(x) u T_{k}(u-\varphi) \leq \int_{\Omega} f T_{k}(u-\varphi),
$$

for every $k>0$ and for every $\varphi$ in $H_{0}^{1}(\Omega) \cap L^{\infty}(\Omega)$.

Theorem 3. Suppose that $f \in L^{1}(\Omega)$. Then there exists an entropy solution $u$ for (2.5).

Proof. We do by approximation; Suppose that $f_{n}=T_{n}(f)$ and by the Lax-Miligram theorem, there exists a weak solution $u_{n}$ for

$$
\begin{cases}-\operatorname{div}\left(A(x) \nabla u_{n}\right)+q(x) u_{n}=f_{n} & \text { in } \Omega \\ u_{n}=0 & \text { on } \partial \Omega .\end{cases}
$$

Let $k>0$. Taking $T_{k}\left(u_{n}\right)$ as test function and using of lemma 2,

$$
\begin{aligned}
\alpha \int_{\Omega}\left|\nabla T_{k}\left(u_{n}\right)\right|^{2} & \leq \int_{\Omega} A(x) \nabla u_{n} \cdot \nabla T_{k}\left(u_{n}\right)+\int_{\Omega} q(x) u_{n} T_{k}\left(u_{n}\right) \\
& =\int_{\Omega} f_{n} T_{k}\left(u_{n}\right) \leq k\|f\|_{L^{1}(\Omega)} .
\end{aligned}
$$

Therefore, $\left(T_{k}\left(u_{n}\right)\right)_{n}$ is bounded in $H_{0}^{1}(\Omega)$ for a fixed $k$. This implies that there exists a function $v_{k} \in H_{0}^{1}(\Omega)$ such that, up to subsequences $T_{k}\left(u_{n}\right)$ converges to $v_{k}$ 
weakly in $H_{0}^{1}(\Omega)$ and strongly in $L^{2}(\Omega)$. From lemma 2, one can deduce that

$$
\int_{\Omega}\left|\nabla\left(u_{n}-u_{m}\right)\right|^{q} \leq C_{q}\left\|f_{n}-f_{m}\right\|_{L^{1}(\Omega)}^{q}
$$

and since $\left(f_{n}\right)_{n}$ is a Cauchy sequence in $L^{1}(\Omega)$, so $\left(u_{n}\right)_{n}$ is a Cauchy sequence in $W_{0}^{1, q}(\Omega)$ and then $u_{n}$ converges strongly to a suitable $u \in W_{0}^{1, q}(\Omega)$. For every $q<\frac{N}{N-1}, \nabla u_{n}$ converges to $\nabla u$ almost everywhere in $\Omega$. Thus, $T_{k}\left(u_{n}\right)$ converges strongly to $T_{k}(u)$ in $L^{2}(\Omega)$, and so $v_{k}=T_{k}(u)$. Therefore, by Fatou lemma,

$$
\alpha \int_{\Omega}\left|\nabla T_{k}\left(u_{n}\right)\right|^{2} \leq \liminf _{n \rightarrow+\infty} \alpha \int_{\Omega}\left|\nabla T_{k}\left(u_{n}\right)\right|^{2} \leq k\|f\|_{L^{1}(\Omega)},
$$

which implies that $u$ belongs to $\tau_{0}^{1,2}$. Fix $k>0, \varphi$ in $H_{0}^{1}(\Omega) \cap L^{\infty}(\Omega)$, and $v:=$ $T_{k}\left(u_{n}-\varphi\right)$ as test function in the weak formulation of (2.5). Then

$$
\int_{\Omega} A(x) \nabla u_{n} . \nabla T_{k}\left(u_{n}-\varphi\right)+\int_{\Omega} q(x) u_{n} T_{k}\left(u_{n}-\varphi\right)=\int_{\Omega} f_{n} T_{k}\left(u_{n}-\varphi\right) .
$$

For the right hand side we have $T_{n} \longrightarrow I$ as $n \longrightarrow \infty$ and $f_{n}=T_{n}(f) \longrightarrow f$. Thus, $f_{n} \longrightarrow f$ point wise in $L^{1}(\Omega)$ and $\left|f_{n} T_{k}\left(u_{n}-\varphi\right)\right| \leq 2 k|f|$. Lebesgue theorem implies that

$$
\lim _{n \rightarrow+\infty} \int_{\Omega} f_{n} T_{k}\left(u_{n}-\varphi\right)=\int_{\Omega} f T_{k}(u-\varphi),
$$

while the left hand side can be rewritten as

$$
\begin{gathered}
\int_{\Omega} A(x) \nabla T_{k}\left(u_{n}-\varphi\right) \cdot \nabla T_{k}\left(u_{n}-\varphi\right)+\int_{\Omega} A(x) \nabla \varphi \cdot T_{k}\left(u_{n}-\varphi\right) \\
+\int_{\Omega} q(x) u_{n} T_{k}\left(u_{n}-\varphi\right) .
\end{gathered}
$$

The first term is non-negative, thus, the almost everywhere convergence of $\nabla u_{n}$ to $\nabla u$ follows by Fatou lemma,

$$
\int_{\Omega} A(x) \nabla T_{k}(u-\varphi) . \nabla T_{k}(u-\varphi) \leq \liminf _{n \rightarrow+\infty}\left(\int_{\Omega} A(x) \nabla T_{k}\left(u_{n}-\varphi\right) . \nabla T_{k}\left(u_{n}-\varphi\right) .\right.
$$

For the second, since $u_{n}$ converges to $u$ in $H_{0}^{1}(\Omega)$ so $u_{n}-\varphi$ to $u-\varphi$ in $H_{0}^{1}(\Omega)$, then $T_{k}\left(u_{n}-\varphi\right)$ to $T_{k}(u-\varphi)$ in $\left.H_{0}^{1}(\Omega)\right)$ and since $-\nabla(A(x) \nabla \varphi) \in H_{0}^{1}$

$$
<-\nabla(A(x) \nabla \varphi), T_{k}\left(u_{n}-\varphi\right)>\longrightarrow<-\nabla(A(x) \nabla \varphi), T_{k}(u-\varphi)>
$$

i.e.

For third term since

$$
\int_{\Omega} A(x) \nabla \varphi \cdot T_{k}(u-\varphi)=\lim _{n \rightarrow+\infty} \int_{\Omega} A(x) \nabla \varphi \cdot T_{k}\left(u_{n}-\varphi\right) .
$$

$$
\left|q(x) u_{n} T_{k}\left(u_{n}-\varphi\right)\right| \leq 2 k\|q\|_{\infty}|u|
$$


by Lebesgue dominated convergence theorem

$$
\int_{\Omega} q(x) u T_{k}(u-\varphi)=\lim _{n \rightarrow+\infty} \int_{\Omega} q(x) u_{n} T_{k}\left(u_{n}-\varphi\right) .
$$

Then by cancelling equal terms:

$$
\int_{\Omega} A(x) \nabla u . \nabla T_{k}(u-\varphi)+\int_{\Omega} q(x) u T_{k}(u-\varphi) \leq \int_{\Omega} f T_{k}(u-\varphi),
$$

so $u$ is an entropy solution of (2.5).

Theorem 4. Let $f \in L^{1}(\Omega)$ and $u$ be an entropy solution of (2.5) with datum $f$. Then $u$ belongs to $W_{0}^{1, q}(\Omega)$ for every $q<\frac{N}{N-1}$ and it is a distributional solution for (2.5).

Proof. Taking $\varphi=0$ in (2.8)

$$
\alpha \int_{\Omega}\left|\nabla T_{k}(u)\right|^{2} \leq \int_{\Omega} A(x) \nabla u . \nabla T_{k}(u)+\int_{\Omega} q(x) u T_{k}(u)=\int_{\Omega} f T_{k}(u) \leq k\|f\|_{L^{1}(\Omega)} .
$$

Proof of Theorem 4.1 in [9] shows that $u \in W_{0}^{1, q}(\Omega)$ for every $q<\frac{N}{N-1}$. We now fix $h>0$ and choose $\varphi=T_{h}(u)$ as test function in (2.8). Then

$$
\int_{\Omega} A(x) \nabla u \cdot \nabla T_{k}\left(u-T_{h}(u)\right)+\int_{\Omega} q(x) u T_{k}\left(u-T_{h}(u)\right) \leq \int_{\Omega} f T_{k}\left(u-T_{h}(u)\right) .
$$

Moreover,

$$
T_{k}\left(u-T_{h}(u)\right)= \begin{cases}u-T_{h}(u) & -k \leq u-T_{h}(u) \leq k \\ k & k \leq u-T_{h}(u) \\ -k & u-T_{h}(u) \leq-k\end{cases}
$$

where

$$
u-T_{h}(u)= \begin{cases}0 & -h \leq u \leq h, \\ u-h & h \leq u, \\ u+h & u \leq-h .\end{cases}
$$

Therefore, if $|u| \leq h$, then $T_{k}\left(u-T_{h}(u)\right)=0$. Moreover, if $h-k \leq|u| \leq h+k$, then $T_{k}\left(u-T_{h}(u)\right)=u-T_{h}(u)$. Thus,

$$
\begin{gathered}
\int_{\{h-k \leq|u| \leq h+k\}} A(x) \nabla u . \nabla u+\int_{\{|u| \geq h\}} q(x) u T_{k}\left(u-T_{h}(u)\right) \\
=\int_{\{|u| \geq h\}} f T_{k}\left(u-T_{h}(u)\right) \leq k \int_{\{|u| \geq h\}}|f| .
\end{gathered}
$$

Defining $A_{h}=\{|u| \geq h\}, m\left(A_{h}\right) \longrightarrow 0$ as $h \longrightarrow \infty$ (since $u \in W_{0}^{1,1}(\Omega)$, thus, in $\left.L^{1}(\Omega)\right)$. From $f \in L^{1}(\Omega)$,

$$
\lim _{h \rightarrow+\infty} \int_{\{|u| \geq h\}}|f|=0,
$$


hence by recalling $(2.7)$

$$
\lim _{h \rightarrow+\infty} \int_{\{h-k \leq|u| \leq h+k\}}|\nabla u|^{2}=0 .
$$

For $h>0, \eta$ in $C_{0}^{1}(\Omega)$ and $\varphi=T_{h}(u)-\eta$ as test function in the entropy formulation (2.8), where $k=\|\eta\|_{L^{\infty}(\Omega)}$ then

$$
\int_{\Omega} A(x) \nabla u \cdot \nabla T_{k}\left(u-T_{h}(u)+\eta\right)+\int_{\Omega} q(x) u T_{k}\left(u-T_{h}(u)+\eta\right) \leq \int_{\Omega} f T_{k}\left(u-T_{h}(u)+\eta\right) .
$$

By Lebesgue dominated theorem and choice of $k$

$$
\lim _{h \rightarrow+\infty} \int_{\Omega} f T_{k}\left(u-T_{h}(u)+\eta\right)=\int_{\Omega} f T_{k}(\eta)=\int_{\Omega} f \eta .
$$

For the left hand side, using again the choice of $k$

$$
\begin{aligned}
& \int_{\{|u| \leq h\}}\left(A(x) \nabla u . \nabla T_{k}\left(u-T_{h}(u)+\eta\right)+\int_{\{|u| \leq h\}} q(x) u T_{k}\left(u-T_{h}(u)+\eta\right)\right) \\
+ & \int_{\{|u| \geq h\}}\left(A(x) \nabla u . \nabla T_{k}\left(u-T_{h}(u)+\eta\right)+\int_{\{|u| \geq h\}} q(x) u T_{k}\left(u-T_{h}(u)+\eta\right)\right) .
\end{aligned}
$$

Since $A$ is bounded, so $u \in W_{0}^{1,1}(\Omega)$ and $\eta \in C_{0}^{1}(\Omega)$. For $\{|u| \leq h\}$ we have $T_{k}(u-$ $\left.T_{h}(u)+\eta\right)=T_{k}(\eta)$. Thus, by Lebesgue dominated theorem

$\lim _{h \rightarrow+\infty} \int_{\{|u| \leq h\}} A(x) \nabla u . \nabla T_{k}(\eta)=\lim _{h \rightarrow+\infty} \int_{\{|u| \leq h\}} A(x) \nabla u . \nabla \eta=\int_{\Omega} A(x) \nabla u . \nabla \eta$.

Similarly

$$
\int_{\{|u| \leq h\}} q(x) u T_{k}\left(u-T_{h}(u)+\eta\right)=\int_{\{|u| \leq h\}} q(x) u T_{k}(\eta)=\int_{\{|u| \leq h\}} q(x) u \eta .
$$

Then

$$
\lim _{h \rightarrow+\infty} \int_{\{|u| \leq h\}} q(x) u \eta=\int_{\Omega} q(x) u \eta .
$$

Since

$$
\left\{\left|u-T_{h}(u)+\eta\right| \leq k,|u| \geq h\right\} \subseteq\{h-2 k \leq|u| \leq h+2 k\}
$$

by (2.7) and choice of $k$

$$
\begin{aligned}
& \int_{\{|u| \geq h\}} A(x) \nabla u \cdot \nabla T_{k}\left(u-T_{h}(u)+\eta\right) \leq\left|\int_{\{|u| \geq h\}} A(x) \nabla u . \nabla T_{k}\left(u-T_{h}(u)+\eta\right)\right| \\
\leq & \int_{\{|u| \geq h\}} A(x)|\nabla u| \cdot\left|\nabla T_{k}\left(u-T_{h}(u)+\eta\right)\right| \leq \beta \int_{\{h-2 k \leq|u| \leq h+2 k\}}|\nabla u|(|\nabla u|+|\nabla \eta|) .
\end{aligned}
$$


Thus by (2.9) and Hölder Inequality

$$
\begin{gathered}
\beta \int_{\{h-2 k \leq|u| \leq h+2 k\}}|\nabla u|(|\nabla u|+|\nabla \eta|) \leq \beta \int_{\{h-2 k \leq|u| \leq h+2 k\}}\left(|\nabla u|^{2}+|\nabla u| \cdot|\nabla \eta|\right) \\
\leq \beta\left[\int_{\{h-2 k \leq|u| \leq h+2 k\}}|\nabla u|^{2}\right]^{\frac{1}{2}} \cdot\left[\int_{\{h-2 k \leq|u| \leq h+2 k\}}|\nabla \eta|^{2}\right]^{\frac{1}{2}}=0 .
\end{gathered}
$$

Therefore,

$$
\lim _{h \rightarrow+\infty} \int_{\{|u| \geq h\}}\left(A(x) \nabla u \cdot \nabla T_{k}\left(u-T_{h}(u)+\eta\right)=0\right.
$$

and

$$
\left.\mid q(x) u T_{k}\left(u-T_{h}(u)+\eta\right)\right)\left|\leq k\|q\|_{\infty}\right| u \mid
$$

so $\left.q(x) u T_{k}\left(u-T_{h}(u)+\eta\right)\right) \in L^{1}(\Omega)$. Thus, $\lim _{h \rightarrow+\infty} \int_{\{|u| \geq h\}} k\|q\|_{\infty}|u|=0$ and

$$
\left.\int_{\{|u| \geq h\}} q(x) u T_{k}\left(u-T_{h}(u)+\eta\right)\right)=0
$$

Putting together the results,

$$
\int_{\Omega}(A(x) \nabla u \cdot \nabla \eta+q(x) u \eta) \leq \int_{\Omega} f \eta,
$$

for any $\eta \in C_{0}^{1}(\Omega)$. Exchanging $\eta$ with $-\eta$ we obtain the reverse inequality so that $u$ is a distributional solution of (2.5).

Finally, we would show uniqueness of entropy solution.

Theorem 5. Let $f \in L^{1}(\Omega)$. Then the entropy solution of (2.5) is unique.

Proof. We proceed in three steps.

Step 1 (An entropy solution is a duality solution): Consider $g$ is in $L^{\infty}(\Omega)$ and $v$ is a weak solution of

$$
\begin{cases}-\operatorname{div}\left(A^{*}(x) \nabla v\right)+q(x) v=g & \text { in } \Omega \\ v=0 & \text { on } \partial \Omega .\end{cases}
$$

According to the Stampacchia's theorem [2], $v \in L^{\infty}(\Omega)$. We repeat the proof of Theorem 4. By choosing $\varphi=T_{h}(u)-v$ in the entropy formulation, for $h>0$ and $k=\|v\|_{L^{\infty}(\Omega)}$ :

$$
\int_{\Omega} A(x) \nabla u \cdot \nabla T_{k}\left(u-T_{h}(u)+v\right)+\int_{\Omega} q(x) u T_{k}\left(u-T_{h}(u)+v\right) \leq \int_{\Omega} f T_{k}\left(u-T_{h}(u)+v\right) .
$$

Similar to theorem 4, from Lebesgue dominated theorem and choose of $k$,

$$
\lim _{h \rightarrow+\infty} \int_{\Omega} f T_{k}\left(u-T_{h}(u)+v\right)=\int_{\Omega} f v,
$$


Moreover, the left hand side can be rewritten as

$$
\begin{gathered}
\int_{\{|u| \leq h\}}(A(x) \nabla u . \nabla v+q(x) u v)+\int_{\{|u| \geq h\}} A(x) \nabla u . \nabla T_{k}\left(u-T_{h}(u)+v\right) \\
+\int_{\{|u| \geq h\}} q(x) u T_{k}\left(u-T_{h}(u)+v\right) .
\end{gathered}
$$

For the second and the third term, similar to the proof of Theorem 4 (using (2.9))

$\lim _{h \rightarrow+\infty} \int_{\{|u| \geq h\}} A(x) \nabla u . \nabla T_{k}\left(u-T_{h}(u)+v\right)+\int_{\{|u| \geq h\}} q(x) u T_{k}\left(u-T_{h}(u)+v\right)=0$,

and the first term can be rewritten as

$$
\begin{gathered}
\int_{\{|u| \leq h\}}(A(x) \nabla u \cdot \nabla v+q(x) u v)=\int_{\Omega}\left(A(x) \nabla T_{h}(u) . \nabla v+q(x) T_{h}(u) v\right) \\
=\int_{\Omega}\left(A^{*}(x) \nabla v \cdot \nabla T_{h}(u)+q(x) v T_{h}(u)\right)=\int_{\Omega} g T_{h}(u),
\end{gathered}
$$

since $T_{h}(u) \in H_{0}^{1}(\Omega)$ can be chosen as test function in the problem solved by $v$. Then, by Lebesgue dominated theorem,

$\int_{\{|u| \leq h\}}(A(x) \nabla u . \nabla v+q(x) u v)=\lim _{h \rightarrow+\infty} \int_{\{|u| \leq h\}}(A(x) \nabla u . \nabla v+q(x) u v)=\int_{\Omega} g u$.

Putting together the results, we obtain

$$
\int_{\Omega} g u \leq \int_{\Omega} f v
$$

Exchanging $g$ with $-g$ (and so $v$ with $-v$, by linearity), we obtain the reverse inequality, therefore, $u$ is a duality solution of (2.5).

Step 2 (An entropy solution is a solution obtained by approximation (See [10])): Suppose that $f_{n} \in L^{\infty}(\Omega)$ be a sequence of functions that converges to $f \in L^{1}(\Omega)$, and suppose that $u_{n}$ be the solution of

$$
\begin{cases}-\operatorname{div}\left(A^{*}(x) \nabla u_{n}\right)+q(x) u_{n}=f_{n} & \text { in } \Omega \\ u_{n}=0 & \text { on } \partial \Omega\end{cases}
$$

$u_{n} \in H_{0}^{1}(\Omega) \cap L^{\infty}(\Omega)$, so that $\varphi=u_{n}$ is an admissible choice in the entropy formulation for $u$. Thus,

$$
\int_{\Omega} A(x) \nabla u \cdot \nabla T_{k}\left(u-u_{n}\right)+\int_{\Omega} q(x) u T_{k}\left(u-u_{n}\right) \leq \int_{\Omega} f T_{k}\left(u-u_{n}\right) .
$$

On the other hand, $T_{k}\left(u-u_{n}\right)$ belongs to $H_{0}^{1}(\Omega)$ and so it can be chosen as test function in the weak formulation for $u_{n}$. Then

$$
\int_{\Omega} A(x) \nabla u_{n} \cdot \nabla T_{k}\left(u-u_{n}\right)+\int_{\Omega} q(x) u_{n} T_{k}\left(u-u_{n}\right)=\int_{\Omega} f_{n} T_{k}\left(u-u_{n}\right) .
$$


Thus,

$\int_{\Omega} A(x) \nabla\left(u-u_{n}\right) \cdot \nabla T_{k}\left(u-u_{n}\right)+\int_{\Omega} q(x)\left(u-u_{n}\right) T_{k}\left(u-u_{n}\right) \leq \int_{\Omega}\left(f-f_{n}\right) T_{k}\left(u-u_{n}\right)$.

By (2.7) and lemma 2

$$
\begin{aligned}
\alpha \int_{\Omega}\left|\nabla T_{k}\left(u-u_{n}\right)\right|^{2} \leq \int_{\Omega} A(x) & \nabla\left(u-u_{n}\right) \cdot \nabla T_{k}\left(u-u_{n}\right)+\int_{\Omega} q(x)\left(u-u_{n}\right) T_{k}\left(u-u_{n}\right) \\
& \leq k\left\|\left(f-f_{n}\right)\right\|_{L^{1}(\Omega)} .
\end{aligned}
$$

approaching $n \longrightarrow \infty, T_{k}\left(u-u_{n}\right) \longrightarrow 0$ in $H_{0}^{1}(\Omega)$ and this implies that $u_{n}$ converges to the entropy solution $u$. From solutions obtained by approximation are unique, hence, the entropy solution $u$ is unique.

Step 3 (There exists at most an entropy solution): Here, we follow [2]. Suppose that $u$ and $v$ be two entropy solutions of (2.5), with the same datum $f$, and let $h>k>0$. Then $\varphi=T_{h}(v)$ is admissible in the entropy formulation for $u$ and $\varphi=T_{h}(u)$ is admissible in the entropy formulation for $v$. Thus,

$$
\int_{\Omega} A(x) \nabla u \cdot \nabla T_{k}\left(u-T_{h}(v)\right)+\int_{\Omega} q(x) u T_{k}\left(u-T_{h}(v)\right) \leq \int_{\Omega} f T_{k}\left(u-T_{h}(v)\right),
$$

and

$$
\int_{\Omega} A(x) \nabla v \cdot \nabla T_{k}\left(v-T_{h}(u)\right)+\int_{\Omega} q(x) v T_{k}\left(v-T_{h}(u)\right) \leq \int_{\Omega} f T_{k}\left(v-T_{h}(u)\right) .
$$

Summing these two inequalities,

$$
\begin{gathered}
\int_{\Omega} A(x) \nabla u \cdot \nabla T_{k}\left(u-T_{h}(v)\right)+\int_{\Omega} q(x) u T_{k}\left(u-T_{h}(v)\right) \\
+\int_{\Omega} A(x) \nabla v \cdot \nabla T_{k}\left(v-T_{h}(u)\right)+\int_{\Omega} q(x) v T_{k}\left(v-T_{h}(u)\right),
\end{gathered}
$$

in the left hand side is less than or equal to

$$
\int_{\Omega} f\left(T_{k}\left(u-T_{h}(v)\right)+T_{k}\left(v-T_{h}(u)\right)\right)
$$

in the right hand side. From oddness of $T_{k}(s)$ and Lebesgue dominated theorem,

$$
\lim _{h \rightarrow+\infty} \int_{\Omega} f\left(T_{k}\left(u-T_{h}(v)\right)+T_{k}\left(v-T_{h}(u)\right)\right)=0 .
$$

Hence,

$$
\begin{aligned}
& \limsup _{h \rightarrow+\infty} \int_{\Omega} A(x) \nabla u \cdot \nabla T_{k}\left(u-T_{h}(v)\right)+\int_{\Omega} q(x) u T_{k}\left(u-T_{h}(v)\right) \\
& +\int_{\Omega} A(x) \nabla v \cdot \nabla T_{k}\left(v-T_{h}(u)\right)+\int_{\Omega} q(x) u T_{k}\left(v-T_{h}(u)\right) \leq 0 .
\end{aligned}
$$


For the sake of simplicity we will suppose from now on that $u \geq 0$ and $v \geq 0$, since the proof turns out to be considerably simplified. We refer to [2] for the proof in the general case of changing sign solutions. We set

$$
\Omega=\{u \leq h, v \leq h\} \cup\{u>h, v \leq h\} \cup\{v>h\}=E_{0}^{h} \cup F_{1}^{h} \cup F_{2}^{h},
$$

and

$$
\Omega=\{v \leq h, u \leq h\} \cup\{v>h, u \leq h\} \cup\{u>h\}=E_{0}^{h} \cup F_{3}^{h} \cup F_{4}^{h} .
$$

Then

$$
\begin{gathered}
\int_{E_{0}^{h}} A(x) \nabla u \cdot \nabla T_{k}\left(u-T_{h}(v)\right)+\int_{E_{0}^{h}} q(x) u T_{k}\left(u-T_{h}(v)\right) \\
=\int_{E_{0}^{h}} A(x) \nabla u \cdot \nabla T_{k}(u-v)+\int_{E_{0}^{h}} q(x) u T_{k}(u-v) .
\end{gathered}
$$

Similarly,

$$
\begin{gathered}
\int_{E_{0}^{h}} A(x) \nabla v \cdot \nabla T_{k}\left(v-T_{h}(u)\right)+\int_{E_{0}^{h}} q(x) v T_{k}\left(v-T_{h}(u)\right) \\
=\int_{E_{0}^{h}} A(x) \nabla v \cdot \nabla T_{k}(v-u)+\int_{E_{0}^{h}} q(x) v T_{k}(v-u),
\end{gathered}
$$

On $F_{1}^{h}$,

$$
\int_{F_{1}^{h}} A(x) \nabla u . \nabla T_{k}\left(u-T_{h}(v)\right)=\int_{\{u>h, v \leq h, 0 \leq u-v \leq k\}} A(x) \nabla u . \nabla(u-v) .
$$

On $\{u>h, v \leq h, 0 \leq u-v \leq k\}$ it valid $h<u \leq h+k$ and $h-k<v \leq h$, so

$$
\left|\int_{F_{1}^{h}} A(x) \nabla u \cdot \nabla T_{k}\left(u-T_{h}(v)\right)\right| \leq \beta \int_{\{h<u \leq h+k, h-k<v \leq h\}}|\nabla u||\nabla v| .
$$

By (2.9)

$$
\lim _{h \rightarrow+\infty} \int_{\{h<u \leq h+k\}}|\nabla u|^{2}=0
$$

and

$$
\lim _{h \rightarrow+\infty} \int_{\{h-k<v \leq h\}}|\nabla v|^{2}=0,
$$

Hence, by Hölder inequality

$$
\lim _{h \rightarrow+\infty}\left|\int_{F_{1}^{h}} A(x) \nabla u . \nabla T_{k}\left(u-T_{h}(v)\right)\right|=0 ;
$$

and $\left|q(x) u T_{k}\left(u-T_{h}(v)\right)\right| \leq k\|q\|_{\infty}|u|$, so $q(x) u T_{k}\left(u-T_{h}(v)\right) \in L^{1}(\Omega)$. Thus,

$$
\lim _{h \rightarrow+\infty} \int_{F_{1}^{h}} k\|q\|_{\infty}|u|=0,
$$


hence,

Repeating the same for $F_{3}^{h}$ we have

$$
\int_{F_{1}^{h}} q(x) u T_{k}\left(u-T_{h}(v)\right)=0 .
$$

$$
\lim _{h \rightarrow+\infty}\left|\int_{F_{3}^{h}} A(x) \nabla v \cdot \nabla T_{k}\left(v-T_{h}(u)\right)\right|=0 .
$$

and

$$
\int_{F_{3}^{h}} q(x) v T_{k}\left(v-T_{h}(u)\right)=0
$$

Moreover on $F_{2}^{h}$,

$$
\begin{gathered}
\int_{F_{2}^{h}}\left(A(x) \nabla u \cdot \nabla T_{k}\left(u-T_{h}(v)\right)+q(x) u T_{k}\left(u-T_{h}(v)\right)\right) \\
\left.=\int_{\{v>h, 0 \leq u<h+k\}}(A(x) \nabla u \cdot \nabla u)+q(x) u u\right) \geq 0,
\end{gathered}
$$

and similarly on $F_{4}^{h}$,

$$
\begin{gathered}
\int_{F_{4}^{h}}\left(A(x) \nabla v \cdot \nabla T_{k}\left(v-T_{h}(u)\right)+q(x) v T_{k}\left(v-T_{h}(u)\right)\right) \\
\left.=\int_{\{u>h, 0 \leq v<h+k\}}(A(x) \nabla v \cdot \nabla v)+q(x) v v\right) \geq 0,
\end{gathered}
$$

Putting the results together,

$$
\limsup _{h \rightarrow+\infty} \int_{E_{0}^{h}}\left(A(x) \nabla(u-v) \cdot \nabla T_{k}(u-v)+q(x)(u-v) T_{k}(u-v)\right) \leq 0,
$$

which, by Fatou lemma, implies, from $E_{0}^{h}$ "fills" $\Omega$ as $h \longrightarrow+\infty$,

$$
0 \leq \int_{\Omega}\left(A(x) \nabla(u-v) . \nabla T_{k}(u-v)+q(x)(u-v) T_{k}(u-v)\right) \leq 0,
$$

Using (2.7) and lemma 2 we have $\nabla T_{k}(u-v) \equiv 0$, thus $u=v$.

Remark 2. In special case if

$$
A=\left[\begin{array}{ccccccc}
\alpha_{1} & 0 & 0 & . & . & . & 0 \\
0 & \alpha_{2} & 0 & . & . & . & 0 \\
\cdot & & & & & & \cdot \\
\cdot & & & & & & \cdot \\
\cdot & & & & & & \cdot \\
0 & 0 & . & . & . & 0 & \alpha_{n}
\end{array}\right]
$$

and $q(x)=\lambda$, problem (2.5) can be rewritten 


$$
\left\{\begin{array} { l l } 
{ - \operatorname { d i v } ( A ( x ) \nabla u ) + q ( x ) u = f } & { \text { in } \Omega } \\
{ u = 0 } & { \text { on } \partial \Omega }
\end{array} \Longrightarrow \left\{\begin{array}{ll}
-\Delta_{\alpha} u+\lambda u=f & \text { in } \Omega \\
u=0 & \text { on } \partial \Omega
\end{array}\right.\right.
$$

Then above theorems are satisfied for this problem.

\section{REFERENCES}

[1] M. Badiale and E. Serra, Semilinear elliptic equations for beginners. Existence results via the variational approach. London: Springer, 2011. doi: 10.1007/978-0-85729-227-8.

[2] P. Bénilan, L. Boccardo, T. Gallouët, R. Gariepy, M. Pierre, and J. L. VÁZQUEZ, "An 11-theory of existence and uniqueness of solutions of nonlinear elliptic equations," Annali della Scuola Normale Superiore di Pisa. Classe di scienze, vol. 22, no. 2, pp. 241-273, 1995.

[3] T. Cazenave, "An introduction to semilinear elliptic equations," Editora do Instituto de Matemática, Universidade Federal do Rio de Janeiro, Rio de Janeiro, vol. 164, 2006.

[4] M. Cozzi, "Interior regularity of solutions of non-local equations in Sobolev and Nikol'skii spaces." Ann. Mat. Pura Appl. (4), vol. 196, no. 2, pp. 555-578, 2017, doi: 10.1007/s10231016-0586-3.

[5] S. Gustafson, K. Kang, and T.-P. Tsai, "Interior regularity criteria for suitable weak solutions of the Navier-Stokes equations.” Commun. Math. Phys., vol. 273, no. 1, pp. 161-176, 2007, doi: 10.1007/s00220-007-0214-6.

[6] Y.-H. Lee and I. Sim, "Global bifurcation phenomena for singular one-dimensional $p$-Laplacian." J. Differ. Equations, vol. 229, no. 1, pp. 229-256, 2006, doi: 10.1016/j.jde.2006.03.021.

[7] R. Ma, "Nodal solutions of second-order boundary value problems with superlinear or sublinear nonlinearities." Nonlinear Anal., Theory Methods Appl., Ser. A, Theory Methods, vol. 66, no. 4, pp. 950-961, 2007, doi: 10.1016/j.na.2005.12.036.

[8] R. Ma and B. Thompson, "Multiplicity results for second-order two-point boundary value problems with superlinear or sublinear nonlinearities." J. Math. Anal. Appl., vol. 303, no. 2, pp. 726 735, 2005, doi: 10.1016/j.jmaa.2004.09.002.

[9] L. Orsina, "Elliptic equations with measure data."

[10] M. M. Porzio, “A uniqueness result for monotone elliptic problems." C. R., Math., Acad. Sci. Paris, vol. 337, no. 5, pp. 313-316, 2003, doi: 10.1016/S1631-073X(03)00347-9.

[11] J. Siljander and J. M. Urbano, "On the interior regularity of weak solutions to the 2-D incompressible Euler equations." Calc. Var. Partial Differ. Equ., vol. 56, no. 5, p. 19, 2017, doi: 10.1007/s00526-017-1231-8.

Authors' addresses

\section{Alizadeh}

University of Mazandaran, factuly of Mathematic sciences, Babolsar, Iran

E-mail address: az.mohsen@gmail.com

\section{Alimohammady}

University of Mazandaran, factuly of Mathematic sciences, Babolsar, Iran

E-mail address: amohsen@umz.ac.ir 